\title{
Modulation of human thyrotropin oligosaccharide structures - enhanced proportion of sialylated and terminally galactosylated serum thyrotropin isoforms in subclinical and overt primary hypothyroidism
}

\author{
J Trojan, M Theodoropoulou ${ }^{1}$, K H Usadel, G K Stalla ${ }^{1}$ and \\ L Schaaf ${ }^{1}$ \\ Zentrum der Inneren Medizin, Klinikum der Johann Wolfgang Goethe-Universität, D-60590 Frankfurt a.M., Germany and ${ }^{1}$ Max-Planck-Institut für Psychiatrie, \\ Endokrinologie und Klinische Chemie, D-80804 München, Germany \\ (Requests for offprints should be addressed to L Schaaf, Max-Planck-Institut für Psychiatrie, Kraepelinstr. 10, D-80804 München, Germany)
}

\begin{abstract}
Enhanced sialylation of thyrotropin (TSH) prolongs its metabolic clearance rate and thus increases the hormone's in vivo bioactivity. This has been shown for hypothyroid rats and for recombinant human TSH, but there are few data on the sialylation of human serum TSH. The aim of this work was to further study sialylated human serum $\mathrm{TSH}$, its precursors bearing terminal galactose residues, and the role of pharmacological doses of thyrotropinreleasing hormone (TRH) on their secretion under different degrees of primary hypothyroidism.

We analyzed serum TSH in patients with subclinical $(n=9)$ and overt primary hypothyroidism $(n=13)$ compared with euthyroid individuals $(n=12)$ and human standard pituitary TSH (IRP 80/558). Blood was drawn before and $30 \mathrm{~min}$ after intravenous administration of $200 \mu \mathrm{g}$ TRH, and TSH was purified by immunoaffinity concentration. The content of sialylated (sialo-) TSH and isoforms bearing terminal galactose (Gal-TSH, asialo-Gal-TSH) was measured by Ricinus communis (RCA 120) affinity chromatography in combination with enzymatic cleavage of sialic acid residues. TSH immunoreactivity was measured by an automated second generation TSH immunoassay.

Pituitary TSH contained $16 \cdot 5 \pm 0 \cdot 8 \%$ Gal-TSH. In euthyroid individuals the proportion of Gal-TSH was

$14 \cdot 6 \pm 1 \cdot 9 \%$, whereas TSH in patients with subclinical and overt primary hypothyroidism contained $23 \cdot 9 \pm 3 \cdot 5 \%$ $(P<0.05$ vs euthyroid individuals $)$ and $21 \cdot 1 \pm 1 \cdot 7 \%$ Gal-TSH respectively. The mean ratio of asialo-Gal TSH was $23 \cdot 8 \pm 0.6 \%$ for pituitary $\mathrm{TSH}, 35 \cdot 7 \pm 4 \cdot 2 \%$ in euthyroid individuals, $48 \cdot 0 \pm 3 \cdot 3 \%$ in patients with subclinical, and $61 \cdot 5 \pm 3 \cdot 8 \%(P<0 \cdot 001$ vs euthyroid individuals) in patients with overt primary hypothyroidism. For pituitary TSH the calculated proportion of sialo-TSH was $6 \cdot 5 \pm 0 \cdot 2 \%$, for euthyroid individuals $20 \cdot 3 \pm 2 \cdot 8 \%$, for patients with subclinical hypothyroidism $24 \cdot 1 \pm 3 \cdot 0 \%$, and for patients with overt primary hypothyroidism $40 \cdot 7 \pm 3 \cdot 0 \% \quad(P<0 \cdot 001$ vs euthyroid individuals $)$. The proportions of Gal-TSH, asialo-Gal-TSH, and sialoTSH did not differ significantly before and after TRH administration in the individuals studied.

Our data show that patients with subclinical and overt primary hypothyroidism have a markedly increased proportion of serum TSH isoforms bearing terminal galactose and sialic acid residues, which may represent a mechanism for the further stimulation of thyroid function. Pharmacological doses of TRH cause an increased quantity of TSH to be released, but do not significantly alter the proportion of sialylated or terminally galactosylated TSH isoforms.

Journal of Endocrinology (1998) 158, 359-365
\end{abstract}

\section{Introduction}

Thyrotropin (TSH) is a heterodimer glycoprotein hormone consisting of an $\alpha$-subunit and a $\beta$-subunit. The $\alpha$-subunit has two asparagine-linked oligosaccharide units, whereas the $\beta$-subunit has only one. Due to differential processing of oligosaccharides during TSH biosynthesis, these structures are heterogeneous: they differ in galactose, mannose and fucose content, branching properties and in the degree of sulfation and sialylation. This microheterogeneity accounts for the fact that TSH is secreted not as a single structure but as a set of glycosylation variants. This has been demonstrated for animal TSH, human TSH (hTSH), and recombinant hTSH expressed in mammalian cell lines (Magner 1990, 1994).

The oligosaccharide residues of TSH are important for the association of subunits, secretion, stability, in vitro biological activity, and, most importantly as they influence 
Table 1 Clinical data of euthyroid individuals and patients with subclinical and overt primary hypothyroidism. Data shown are means \pm S.E.M. Normal ranges for TSH, total $\mathrm{T}_{3}$, total $\mathrm{T}_{4}$, and free $\mathrm{T}_{4}$ are given in parentheses

\begin{tabular}{|c|c|c|c|}
\hline & $\begin{array}{l}\text { Euthyroid } \\
\text { individuals }\end{array}$ & $\begin{array}{l}\text { Subclinical } \\
\text { hypothyroidism }\end{array}$ & $\begin{array}{l}\text { Overt primary } \\
\text { hypothyroidism }\end{array}$ \\
\hline Number (female:male) & $12(9: 3)$ & $9(7: 2)$ & $13(10: 3)$ \\
\hline Age (years) & $37 \pm 4$ & $32 \pm 4$ & $46 \pm 1$ \\
\hline \multicolumn{4}{|l|}{ Cause of hypothyroidism } \\
\hline Chronic autoimmune thyroiditis* & & 5 & 6 \\
\hline Posttherapeutic $^{\mathrm{a}}$ & & 2 & 5 \\
\hline Unknown & & 2 & 2 \\
\hline TSH $(0 \cdot 4-5 \cdot 5 \mathrm{mIE} / \mathrm{l})$ & $11 \cdot 8 \pm 0 \cdot 3$ & $19 \cdot 5 \pm 4 \cdot 2$ & $54 \cdot 4 \pm 11 \cdot 3$ \\
\hline TRH-TSH ${ }^{\mathrm{b}}(\mathrm{mlE} / \mathrm{l})$ & $12 \cdot 8 \pm 1 \cdot 5$ & $79 \cdot 4 \pm 22 \cdot 4$ & $129 \cdot 8 \pm 23 \cdot 8$ \\
\hline Total $\mathrm{T}_{3}(0 \cdot 7-2 \cdot 0 \mathrm{ng} / \mathrm{ml})$ & $1 \cdot 3 \pm 0 \cdot 1$ & $1 \cdot 3 \pm 0 \cdot 1$ & $0 \cdot 6 \pm 0 \cdot 2$ \\
\hline Total $\mathrm{T}_{4}(5 \cdot 0-12 \cdot 0 \mu \mathrm{g} / \mathrm{dl})$ & $7 \cdot 4 \pm 0 \cdot 5$ & $6 \cdot 5 \pm 0 \cdot 4$ & $2 \cdot 8 \pm 0 \cdot 6$ \\
\hline Free $\mathrm{T}_{4}(0 \cdot 8-2 \cdot 0 \mathrm{ng} / \mathrm{ml})$ & $1 \cdot 0 \pm 0 \cdot 1$ & $0 \cdot 9 \pm 0 \cdot 2$ & $0 \cdot 4 \pm 0 \cdot 1$ \\
\hline
\end{tabular}

the hormone's metabolic clearance rate, the in vivo bioactivity (Grossmann et al. 1997a). TSH isoforms bearing terminal sialic acid (sialo-TSH) have a decreased metabolic clearance rate leading to an increased plasma circulation time and therefore to an enhanced biological activity (Szkudlinski et al. 1995a). In glycoprotein hormones, sialic acid is preferentially bound to precursors bearing terminal galactose residues (Baenziger \& Green 1988). In addition to an increased quantity of TSH an enhanced sialylation of TSH has been observed in hypothyroid animals, which is believed to increase further the in vivo bioactivity of TSH (Magner 1990). The enzymes responsible for the coupling of terminal galactose and sialylation of TSH, $\beta-1,4-$ galactosyltransferase and sialyltransferases, are more strongly expressed in thyrotropes of hypothyroid rats than in euthyroid controls (Helton \& Magner 1994a,b).

A major difficulty in analyzing the carbohydrate structures of human serum glycoprotein hormones is the limited amount of available material per individual. Therefore, direct analysis of the carbohydrate content of TSH, which constitutes $20-35 \%$ of its weight, is not possible. To overcome this problem, lectins are widely used to characterize the oligosaccharides of serum glycoproteins by affinity chromatography (Gesundheit et al. 1987, Kobata \& Endo 1992). Ricinus communis (RCA 120) is a lectin that binds glycoproteins with exposed galactose (Gal) residues, but does not bind to $\mathrm{N}$-acetylgalactosamine or sulfate (Lin \& Li 1980). Neuraminidase cleaves sialic acid from glycoproteins, exposing more $\mathrm{Gal}$ residues and causing an increase in ricin binding (Miura et al. 1989). To date, there have only been two studies analyzing the degree of sialo-TSH in patients with overt primary hypothyroidism (Miura et al. 1989, Papandreou et al. 1993). Furthermore, there is no study on TSH sialylation in patients with subclinical hypothyroidism. In a recent study, Schaaf et al. (1995) used the lectin Lentil to further characterize the content of core fucose-bearing TSH isoforms in euthyroid and subclinical hypothyroid patients. They demonstrated a substantial shift in core fucose-bearing TSH isoforms in subclinical hypothyroidism. Magner et al. (1992a) have shown that in patients with TSH-secreting pituitary adenomas the degree of core fucosylated TSH tends to be high when sialylation is low and vice versa.

The aim of this work was to further study serum sialo-TSH, its precursors bearing terminal galactose, and the role of pharmacological doses of thyrotropin-releasing hormone (TRH) on the secretion of these TSH isoforms in patients with different degrees of primary hypothyroidism.

\section{Materials and Methods}

\section{Subjects}

Serum samples were obtained from euthyroid volunteers ( $n=12$, 9 females, 3 males, aged $37 \pm 4$ years), patients with subclinical $(n=13,7$ females, 2 males, aged $34 \pm 4$ years) and overt primary hypothyroidism ( $n=12,10$ females, 3 males, aged $46 \pm 1$ years). The hypothyroid metabolic state was caused by chronic autoimmune thyroiditis in five patients with subclinical and in six patients with overt primary hypothyroidism. Two patients with subclinical hypothyroidism and five patients with overt primary hypothyroidism had undergone radiotherapy or thyroid surgery for Grave's disease. The patients' profiles are summarised in Table 1. Blood was drawn before and $30 \mathrm{~min}$ after intravenous (i.v.) administration of $200 \mu \mathrm{g}$ TRH (Relefact, Hoechst, Frankfurt, Germany). In addition, human standard pituitary TSH 
(IRP 80/558, National Institute for Biological Standards and Controls, Potters Bar, Herts, UK) was studied. Written informed consent was obtained from each patient and the investigation was approved by the local ethical committee.

\section{Immunoaffinity purification of human serum TSH}

TSH from serum samples $(10 \mathrm{ml})$ was purified with anti-hTSH antibody-coated polystyrene tubes (Schaaf et al. 1995). In brief, $0.5 \mathrm{ml}$ serum was incubated per tube at room temperature for $4 \mathrm{~h}$. The supernatant was then decanted and antibody-bound TSH eluted with $0.2 \mathrm{M}$ glycine- $\mathrm{HCl}(\mathrm{pH} 2 \cdot 2)$. Aliquots were diluted with $0.01 \%$ bovine serum albumin, pooled, and concentrated on the Centriprep ultrafiltration system (Amicon, Witten, Germany).

\section{Ricinus communis (RCA 120) affinity chromatography}

For cleavage of sialic acid residues, a TSH aliquot was incubated with $10 \mathrm{mIE}$ neuraminidase type X (Sigma, Deisenhofen, Germany), dissolved in citrate buffer $(100 \mathrm{mmol} / \mathrm{l}, \mathrm{pH} 6 \cdot 6)$, for $4 \mathrm{~h}$ at $37^{\circ} \mathrm{C}$. Before Ricinus communis affinity chromatography (RCA 120) (Sigma) native TSH and neuraminidase-treated TSH were diluted with $200 \mu \mathrm{l}$ phosphate-buffered saline (PBS) and the

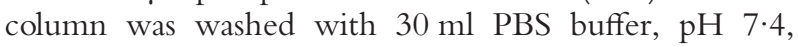
containing natrium azide $(1 \mathrm{~g} / \mathrm{l})$ and bovine serum albu$\min (1 \mathrm{~g} / \mathrm{l})$. Unbound material was washed off the column with $10 \mathrm{ml}$ PBS buffer and bound material was subsequently eluted with PBS buffer, containing $\alpha$-D-galactose ( $200 \mathrm{mmol} / \mathrm{l})$. TSH was measured in $1 \mathrm{ml}$ fractions with an automated TSH immunoassay. The content of TSH isoforms bearing exposed, terminal galactose residues (GalTSH) was calculated as the binding ratio of native TSH. The content of asialo-Gal-TSH was calculated as the binding ratio of neuraminidase-treated TSH. The binding difference of native and digested TSH represents the degree of sialo-TSH (Gesundheit et al. 1986).

\section{Immunometrical assays}

Serum levels of total tri-iodothyronine $\left(\mathrm{T}_{3}\right)$, total thyroxine $\left(\mathrm{T}_{4}\right)$, free thyroxine $\left(\mathrm{FT}_{4}\right)$, and anti-thyroidal peroxidase antibodies (anti-TPO) were determined by standard assays. TSH in each sample was measured with an automated sandwich chemiluminometric assay (ACS TSH, Chiron Diagnostics, Fernwald, Germany), based on a combination of coupled polyclonal anti-hTSH antibody (sheep) and fluorescence-labeled monoclonal anti-hTSH antibody (mouse). The analytical sensitivity of this assay was $0.015 \mathrm{mIE} / 1$ and the cross-reactivity with human choriogonadotropin was less than $1 \%$. To rule out the possibility that this assay does not recognise all TSH isoforms, TSH isoforms isolated by lectin affinity chroma- tography were diluted and measured with another highly sensitive TSH assay (CoTube TSH IRMA, Biorad, München, Germany; analytical sensitivity 0.02 mIE/l). This assay is based on a combination of coupled polyclonal anti-hTSH antibody (mouse) and ${ }^{125}$ I-labeled monoclonal anti-hTSH antibody (mouse). TSH values did not differ significantly in the two assays. For the ACS TSH assay, intra- and interassay precisions were 3.8 and $6.6 \%$ respectively.

\section{Statistical analysis}

Unless otherwise stated data are shown as means \pm S.E.M. Statistical analysis was performed with the Student's $t$-test and significance was assigned to two-tailed $P$ values less than 0.05 vs TSH from euthyroid individuals, before or $30 \mathrm{~min}$ after i.v. administration of $200 \mu \mathrm{g}$ TRH.

\section{Results}

Serum TSH, purified from blood samples obtained before and $30 \mathrm{~min}$ after i.v. administration of $200 \mu \mathrm{g} \mathrm{TRH}$ (TRH-TSH), was analyzed from patients with subclinical and overt primary hypothyroidism and euthyroid individuals. The recovery for basal TSH by the immunoaffinity purification method was $74 \cdot 4 \pm 6.5 \%$ and for TRHreleased TSH it was $78 \cdot 6 \pm 7 \cdot 4 \%$. The reproducibility of TSH binding to Ricinus communis was confirmed by nine independent runs using 5-35 $\mu \mathrm{IE}$ pituitary TSH. The recovery of native TSH was $86 \cdot 4 \pm 1 \cdot 2 \%$ and that of neuraminidase-treated TSH was $80 \cdot 3 \pm 3 \cdot 6 \%$.

\section{Ricin binding of native TSH}

TSH oligosaccharide residues were further characterized with Ricinus communis affinity chromatography. As measured by ricin binding of native TSH, human pituitary TSH contained $16 \cdot 5 \pm 0 \cdot 8 \%$ Gal-TSH. Serum TSH from euthyroid individuals contained 14.6 $\pm 1 \cdot 9 \%$ Gal-TSH, whereas the proportion of serum Gal-TSH in patients with subclinical and overt primary hypothyroidism was $23.9 \pm 3.5 \%(P<0.05$ vs euthyroid individuals $)$ and $21 \cdot 1 \pm 1 \cdot 7 \%$ respectively (Fig. 1 ). After TRH stimulation Gal-TSH represented $14 \cdot 2 \pm 1.9 \%$ of total serum TSH in euthyroid individuals, $23 \cdot 0 \pm 2 \cdot 7 \%$ in patients with subclinical, and $19 \cdot 0 \pm 1 \cdot 6 \%$ in patients with overt primary hypothyroidism. These values did not differ significantly from the proportions of basal TSH (Fig. 2).

\section{Ricin binding of neuraminidase-treated TSH}

Ricin-binding of TSH from all patients studied and from human pituitary extracts was increased after digestion with the sialic acid residue-cleaving enzyme neuraminidase $(P<0 \cdot 05$ vs native TSH). After neuraminidase digestion, 
(a)

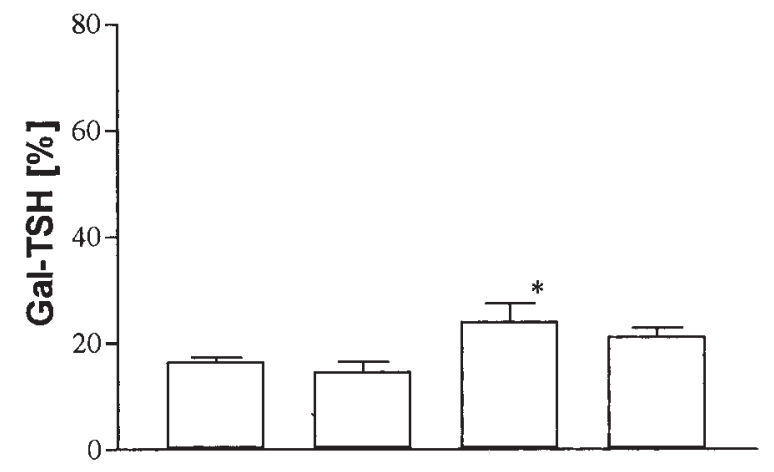

(b)

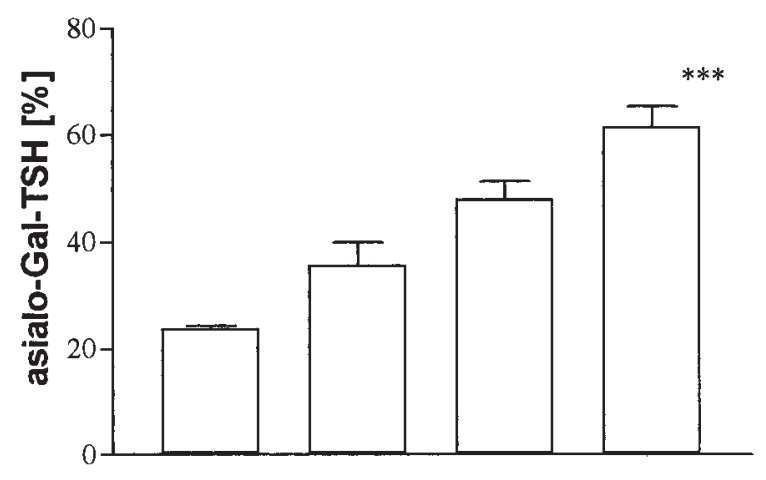

(c)



Figure 1 Ricinus communis (RCA 120) affinity chromatography of native pituitary standard TSH (IRP-TSH) and basal serum TSH in euthyroid (EU, $n=12)$, subclinical $(\mathrm{SPH}, n=9)$ and overt primary hypothyroid patients $(\mathrm{OPH}, n=13)$. (a) Analysis of the ratio of terminally galactosylated (Gal)-TSH, (b) of neuraminidase-treated, terminally galactosylated (asialo-Gal)-TSH, and (c) determination of sialylated (sialo)-TSH. TSH was measured in duplicate with the ACS TSH assay. Data are expressed as means \pm S.E.M. binding to Ricinus Communis. For pituitary TSH the means \pm S.E.M. binding of three independent runs are shown. ${ }^{*} P<0 \cdot 05,{ }^{* *} P<0 \cdot 001$ vs TSH from euthyroid individuals (Student's t-test). (a)

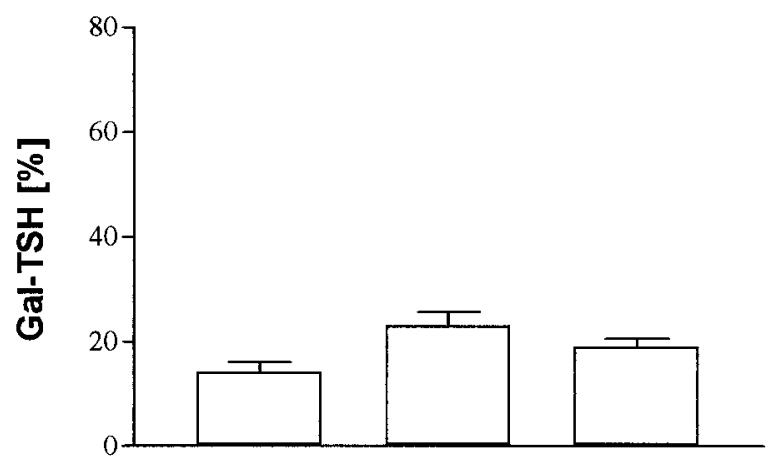

(b)

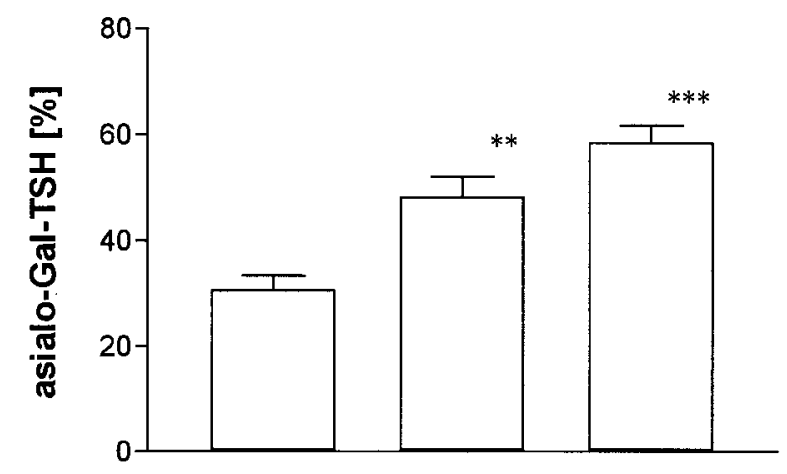

(c)

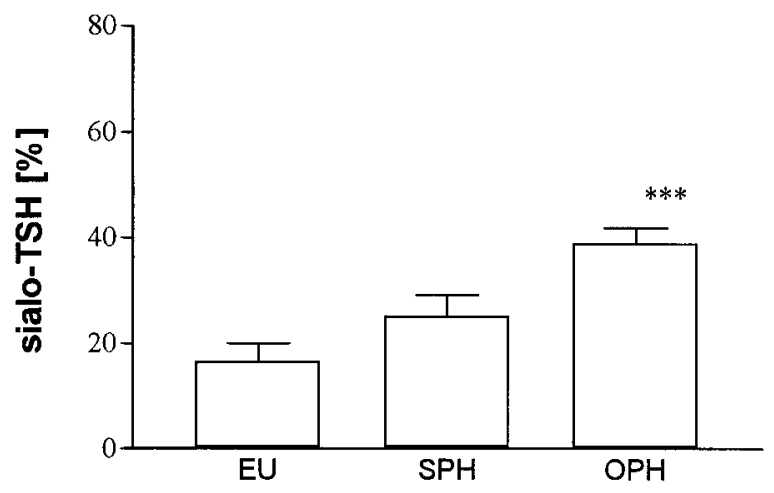

Figure 2 Ricinus communis (RCA 120) affinity chromatography of TRH-released serum TSH in euthyroid individuals (EU, $n=12$ ) and patients with subclinical $(\mathrm{SPH}, n=9)$ and overt primary hypothyroidism $(\mathrm{OPH}, n=13)$. (a) Analysis of the ratio of terminally galactosylated (Gal)-TSH, (b) of neuraminidase-treated, terminally galactosylated (asialo-Gal)-TSH, and (c) determination of sialylated (sialo)-TSH. Serum was obtained 30 min after i.v. administration of $200 \mu \mathrm{g}$ TRH. TSH was measured in duplicate with the ACS TSH assay. Data are expressed as means \pm S.E.M. binding to Ricinus communis. ${ }^{*} P<0 \cdot 01,{ }^{* * *} P<0 \cdot 001$ vs TSH from euthyroid individuals (Student's t-test). 
human pituitary TSH contained $23 \cdot 8 \pm 0 \cdot 6 \%$ asialo-Gal$\mathrm{TSH}$, whereas this proportion was $35 \cdot 7 \pm 4 \cdot 2 \%$ for $\mathrm{TSH}$ in euthyroid individuals. After neuraminidase treatment the mean binding of TSH from subclinical and overt hypothyroid patients was $48 \cdot 0 \pm 3 \cdot 3 \%$ and $61 \cdot 5 \pm 3 \cdot 8 \%$ respectively. Compared with euthyroid individuals the proportion of asialo-TSH in patients with overt primary hypothyroidism was significantly increased $(P<0 \cdot 001)$ (Fig. 1). After administration of TRH the proportion of asialo-TSH was $30 \cdot 6 \pm 2 \cdot 7 \%$ in euthyroid individuals, $48 \cdot 3 \pm 3 \cdot 7 \%$ in patients with subclinical hypothyroidism $(P<0 \cdot 01$ vs euthyroid individuals), and $58 \cdot 6 \pm 3 \cdot 1 \%$ in patients with overt primary hypothyroidism $(P<0.001$ vs euthyroid individuals) (Fig. 2).

\section{Determination of sialo-TSH}

The proportion of sialo-TSH was calculated from the binding difference of native and neuraminidase-treated TSH. For pituitary TSH the calculated proportion of sialo-TSH was $6 \cdot 5 \pm 0 \cdot 2 \%$. TSH in euthyroid patients contained $20 \cdot 3 \pm 2 \cdot 8 \%$ sialo-TSH before and $16 \cdot 6 \pm 3 \cdot 4 \%$ $30 \mathrm{~min}$ after i.v. administration of TRH. The mean proportion of sialo-TSH in patients with subclinical hypothyroidism was $24 \cdot 1 \pm 3 \cdot 0 \%$, whereas this proportion was $40 \cdot 7 \pm 3 \cdot 0 \%$ in overt primary hypothyroid patients $(P<0.001$ vs euthyroid individuals) (Fig. 1). After TRH administration the proportion of sialo-TSH in patients with subclinical and overt primary hypothyroidism was $25 \cdot 1 \pm 4 \cdot 0 \%$ and $38.9 \pm 2 \cdot 9 \% \quad(P<0 \cdot 001$ vs euthyroid individuals) respectively (Fig. 2).

\section{Discussion}

Sialylation of human serum TSH, its precursors bearing terminal galactose residues, and the role of pharmacological doses of TRH on their secretion have not yet been analyzed sufficiently. We studied serum TSH in patients with subclinical and overt primary hypothyroidism because sialylation of TSH seems to have an important impact on the hormone's in vivo bioactivity due to protection from carbohydrate-specific hepatic receptormediated clearance mechanisms (Szkudlinski et al. 1995b). In addition, sialylation is a major factor affecting the charge heterogeneity of TSH (Szkudlinski et al. 1993), which is believed to cause steric changes in the three-dimensional structure of TSH and thus influence the hormone's ability to stimulate the TSH receptor. In accordance with this, it has been demonstrated that certain human TSH glycosylation variants activate inosital phosphate and cAMP signal transduction pathways to different degrees (Schaaf et al. 1997).

As direct analysis of the carbohydrate content of individual human serum TSH, as described for human pituitary glycoprotein hormones (Baenziger \& Green 1988), is not possible we chose Ricinus communis, a lectin that binds oligosaccharides with exposed, terminal galactose. Ricinus communis affinity chromatography in combination with neuraminidase, an enzyme that cleaves sialic acid residues from oligosaccharide chains, is helpful in studying the degree of TSH sialylation (Gesundheit et al. 1986). In order to compare our results with previously published work, we have chosen identical conditions to cleave sialic acid residues from TSH oligosaccharides by means of neuraminidase treatment (Miura et al. 1989, Magner et al. 1992a, Papandreou et al. 1993). In addition, we feel that the chosen digestion time of $4 \mathrm{~h}$ might overcome incomplete cleavage of sialic acid due to possible different kinetics concerning the hydrolyzation of $\alpha 2,3-$ and $\alpha 2,6-$ linked sialic acid residues of TSH. TSH was measured with a highly sensitive immunoassay that does not discriminate between different TSH isoforms (data not shown). TSH values measured with two TSH assays, based on different anti-hTSH antibodies, were similar. Using these methods we can report the following results: compared with euthyroid individuals, patients with subclinical hypothyroidism have an enhanced proportion of serum Gal-TSH, but not of serum sialo-TSH. However after TRH stimulation, the proportion of serum sialo-TSH was also significantly increased in subclinical hypothyroid individuals. In contrast, patients with overt primary hypothyroidism had a markedly increased proportion of both serum Gal-TSH and sialo-TSH before and after TRH administration. Human pituitary-derived TSH, representing a mixture of more or less mature isoforms, contained a lower degree of both Gal- and sialo-TSH than serum TSH in all individuals studied.

During the post-translational processing of TSH its oligosaccharide chains are added and modified. Although a quite late event in this pathway, the addition of Gal versus $\mathrm{N}$-acetylgalactosamine is a key branching point: oligosaccharide chains containing exposed galactose residues may be further modified by attaching terminal sialic acid, whereas $\mathrm{N}$-acetylgalactosamine residues may become sulfated (Baenziger \& Green 1988). The cellular mechanisms causing an increased proportion of secreted Galand sialo-TSH in hypothyroid rats were examined by Helton and Magner (1994a,b). Using in situ hybridisation they demonstrated that the levels of messenger RNA of the Golgi enzymes involved - namely $\beta$ 1,4-galactosyltransferase and $\beta$-galactoside $\alpha-2,6-$ sialyltransferase - are elevated. To date, the expression of these transferases has not been studied in subclinical hypothyroidism or mild hypothyroidism. Interestingly, we found an enhanced proportion of serum Gal-TSH, but not of serum sialo-TSH, in subclinical hypothyroid patients. Therefore, we speculate that the responsible enzyme $\beta-1,4$-galactosyltransferase is already activated in subclinical hypothyroidism. Assuming that the increased proportion of TSH isoforms with terminal galactose implies enhanced TSH sialylation and that the activation of 
galactosyltransferase is followed by the activation of sialyltransferases, the presence of an increased proportion of serum Gal-TSH could turn out to be a marker for the progression of hypothyroidism. Our findings are in accordance with others who have demonstrated an increased proportion of serum sialo-TSH in hypothyroidism compared with euthyroid controls (Miura et al. 1989, Papandreou et al. 1993). Miura et al. (1989) found no correlation between the sialo-TSH proportion and serum TSH level, but patients with hypothyroidism ongoing for more than 1 year had a higher sialo-TSH proportion than those who had been hypothyroid for less than 3 months. Recently, Magner and co-workers (1997) reported a case of decreased TSH sialylation in a patient with euthyroid sick syndrome, which may account for the altered TSH bioactivity that has been described in such patients.

In euthyroid individuals, the i.v. administration of TRH releases TSH containing more core fucose residues than basal TSH (Magner et al. 1992b). We found no statistical difference in TRH-released Gal-TSH, asialo Gal-TSH, and sialo-TSH in euthyroid individuals or in patients with subclinical or overt hypothyroidism compared with basal TSH. In a recent publication, Schaaf et al. (1995) showed an enhanced core fucosylation of TRH-released TSH in patients with subclinical hypothyroidism compared with euthyroid individuals. In the present study, we found a substantial increase in the proportion of sialo-TSH in patients with overt hypothyroidism, but not in those with subclinical hypothyroidism. This might explain the findings of Schaaf et al. (1995) who failed to show a difference in the net charge between serum TSH in euthyroid and subclinical hypothyroid individuals, suggesting a similar proportion of negatively charged TSH isoforms. Grossmann and colleagues (1997b) demonstrated that recombinant TSH, expressed in insect cells, lacks complex-type oligosaccharides terminating with sialic acid but contains predominantly high mannose-type oligosaccharides. This resulted in an increased in vitro bioactivity but, due to rapid metabolic clearance, in a much lower in vivo activity compared with recombinant TSH, expressed in Chinese hamster ovary cells (Szkudlinski et al. 1993). These data support the hypothesis that glycosylation of recombinant hTSH is dependent on the cell system used and is most likely due to different expression of Golgi enzymes. In vivo biological activity of TSH is crucial, especially for the use of recombinant human TSH in stimulating radioiodine uptake for radioactive iodine scanning in patients with thyroid cancer (Ladenson et al. 1997).

In summary, the present study confirmed an enhanced proportion of terminally sialylated serum TSH isoforms in patients with overt primary hypothyroidism. In contrast to overt hypothyroidism, patients with subclinical hypothyroidism have an unchanged proportion of sialylated TSH isoforms, but the proportion of TSH isoforms with terminal galactose, representing precursors of sialo-TSH, was enhanced. Pharmacological doses of TRH cause an increased quantity of TSH to be released, but do not significantly alter the proportion of sialylated or terminally galactosylated TSH isoforms. The physiological roles of TSH isoforms are not precisely understood yet, but the modulation of TSH heterogeneity by regulation of glycosyltransferases may be a mechanism to stimulate further thyroid function.

\section{Acknowledgements}

We thank Dr A Bergmann (BRAHMS, Berlin, Germany) for supplying coated tubes and Dr M Butz (Chiron Diagnostics, Fernwald, Germany) for supplying ACS TSH assay kits. This study was supported by grants from the Deutsche Forschungsgemeinschaft (DFG) Scha 708 (1-1) and J Trojan was supported by a fellowship from the Boehringer Ingelheim Fonds, Stuttgart, Germany.

\section{References}

Baenziger JU \& Green ED 1988 Pituitary glycoprotein hormone oligosaccharides: structure, synthesis and function of the asparagine-linked oligosaccharides on lutropin, follitropin and thyrotropin. Biochimica et Biophysica Acta 947 287-306.

Gesundheit N, Magner JA, Chen T \& Weintraub BD 1986 Differential sulfation and sialylation of secreted mouse thyrotropin (TSH) subunits: regulation by TSH-releasing hormone. Endocrinology 119 455-463.

Gesundheit N, Fink DL, Silverman LA \& Weintraub BD 1987 Effect of thyrotropin-releasing hormone on the carbohydrate structure of secreted mouse thyrotropin. Analysis by lectin affinity chromatography. Journal of Biological Chemistry 262 5197-5203.

Grossmann M, Weintraub BD \& Szkudlinski MW 1997a Novel insights into the molecular mechanisms of human thyrotropin action: structural, physiological, and therapeutic implications for the glycoprotein hormone family. Endocrine Reviews 18 476-501.

Grossmann M, Wong R, Teh NG, Tropea JE, East-Palmer J, Weintraub BD \& Szkudlinski MW $1997 b$ Expression of biologically active human thyrotropin (hTSH) in a baculovirus system: effect of insect cell glycosylation on hTSH activity in vitro and in vivo. Endocrinology 138 92-100.

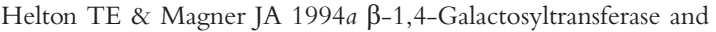
$\alpha$-mannosidase-II messenger ribonucleic acid levels increase with different kinetics in thyrotrophs of hypothyroid mice. Endocrinology 135 1980-1985.

Helton TE \& Magner JA $1994 b$ Sialyltransferase messenger ribonucleic acid increases in thyrotrophs of hypothyroid mice: an in situ hybridization study. Endocrinology 134 2347-2353.

Kobata A \& Endo T 1992 Immobilized lectin columns: useful tools for the fractionation and structural analysis of oligosaccharides. Journal of Chromatography 597 111-122.

Ladenson PW, Braverman LE, Mazzaferri EL, Brucker-Davis F, Cooper DS, Garber JR, Wondisford FE, Davies TF, DeGroot LJ, Daniels GH, Ross DS \& Weintraub BD 1997 Comparison of administration of recombinant human thyrotropin with withdrawal of thyroid hormone for radioactive iodine scanning in patients with thyroid carcinoma. New England Journal of Medicine 337 888-896.

Lin TT \& Li SS 1980 Purification and physiochemical properties of ricins and agglutinins from Ricinus communis. European Journal of Biochemistry 105 453-459. 
Magner JA 1990 Thyroid-stimulating hormone: biosynthesis, cell biology, and bioactivity. Endocrine Reviews 11 354-385.

Magner JA 1994 Biosynthesis, cell biology, and bioactivity of thyroid-stimulating hormone: update 1994. Endocrine Reviews 15 $55-60$.

Magner JA, Klibanski A, Fein H, Smallridge R, Blackard W, Young WJ, Ferriss JB, Murphy D, Kane J \& Rubin D 1992a Ricin and lentil lectin-affinity chromatography reveals oligosaccharide heterogeneity of thyrotropin secreted by 12 human pituitary tumors. Metabolism 41 1009-1015.

Magner JA, Kane J \& Chou ET $1992 b$ Intravenous thyrotropin (TSH)-releasing hormone releases human TSH that is structurally different from basal TSH. Journal of Clinical Endocrinology and Metabolism 74 1306-1311.

Magner J, Roy P, Fainter L, Barnard V \& Fletcher P Jr 1997 Transiently decreased sialylation of thyrotropin (TSH) in a patient with the euthyroid sick syndrome. Thyroid 7 55-61.

Miura Y, Perkel VS, Papenberg KA, Johnson MJ \& Magner JA 1989 Concanavalin-A, lentil, and ricin lectin affinity binding characteristics of human thyrotropin: differences in the sialylation of thyrotropin in sera of euthyroid, primary, and central hypothyroid patients. Journal of Clinical Endocrinology and Metabolism 69 985-995.

Papandreou MJ, Persani L, Asteria C, Ronin C \& Beck-Peccoz P 1993 Variable carbohydrate structures of circulating thyrotropin as studied by lectin affinity chromatography in different clinical conditions. Journal of Clinical Endocrinology and Metabolism 77 393-398.
Schaaf L, Trojan J, Helton TE, Usadel KH \& Magner JA 1995 Serum thyrotropin (TSH) heterogeneity in euthyroid individuals and patients with subclinical hypothyroidism: the core fucose content of TSH-releasing hormone-released TSH is altered but not the net charge of TSH. Journal of Endocrinology 144 561-567.

Schaaf L, Leiprecht A, Saji M, Huebner U, Usadel KH \& Kohn LD 1997 Glycosylation variants of human TSH selectively activate signal transduction pathways. Molecular and Cellular Endocrinology 132 185-194.

Szkudlinski MW, Thotakura NR, Bucci I, Joshi LR, Tsai A, EastPalmer J, Shiloach J \& Weintraub BD 1993 Purification and characterization of recombinant human thyrotropin (TSH) isoforms produced by Chinese hamster ovary cells: the role of sialylation and sulfation in TSH bioactivity. Endocrinology 133 1490-1503.

Szkudlinski MW, Thotakura NR \& Weintraub BD 1995a Subunit-specific functions of $\mathrm{N}$-linked oligosaccharides in human thyrotropin: role of terminal residues of alpha- and beta-subunit oligosaccharides in metabolic clearance and bioactivity. Proceedings of the National Academy of Sciences of the USA 92 9062-9066.

Szkudlinski MW, Thotakura NR, Tropea JE, Grossmann M \& Weintraub BD $1995 b$ Asparagine-linked oligosaccharide structures determine clearance and organ distribution of pituitary and recombinant thyrotropins (TSH). Endocrinology 136 3325-3330.

Received 20 January 1998

Accepted 29 April 1998 